\title{
Using $\mathbf{H}_{2}$ Emission to Study the Fast Wind in Proto-Planetary Nebulae
}

\author{
Bruce J. Hrivnak ${ }^{1}$, Nathan Smith ${ }^{2}$, Kate Y.L. Su ${ }^{3}$, \\ Douglas M. Kelly ${ }^{3}$, Sun Kwok ${ }^{4}$ and Raghvendra Sahai ${ }^{5}$ \\ ${ }^{1}$ Department of Physics and Astronomy, Valparaiso University, Valparaiso, IN 46383, USA \\ email: bruce.hrivnak@valpo.edu \\ ${ }^{2}$ CASA, University of Colorado, Boulder, CO 80309, USA \\ ${ }^{3}$ Steward Observatory, University of Arizona, Tucson, AZ 85721, USA \\ ${ }^{4}$ Department of Physics, University of Hong Kong, Hong Kong, China \\ ${ }^{5}$ JPL/Caltech, 4800 Oak Grove Drive, Pasadena, CA 91109, USA
}

\begin{abstract}
We present the results of a high-resolution $\mathrm{H}_{2}$ imaging and spectroscopic study of four bipolar proto-planetary nebulae: IRAS 16594-4656, Hen 3-401, Rob 22, and IRAS 17150-3224. These reveal the locations and kinematics of the $\mathrm{H}_{2}$ emission and the shaping of the nebulae by their collimated fast winds.
\end{abstract}

Keywords. circumstellar matter - planetary nebulae: general - stars: AGB and post-AGB

\section{Introduction}

Planetary nebulae and their immediate precursors, the transitional proto-planetary nebulae (PPNe), are observed to have a variety of morphologies: circular, elliptical, bipolar, point-symmetric. The shaping of bipolar PPNe is thought to occur as a fast wind from the central star interacts with a circumstellar envelope containing a latitudinal density gradient. Thus collisionally-excited $\mathrm{H}_{2}$ emission in the nebulae can provide a tracer of the fast wind as it interacts with the remnant AGB mass loss which forms the circumstellar envelope. We have carried out a study of the location and kinematics of the $\mathrm{H}_{2}$ emission in four bipolar PPNe.

\section{Observations}

High-resolution $2 \mu \mathrm{m}$ images were obtained with NICMOS on HST. Images with the F212N filter include the $\mathrm{H}_{2}$ 1-0 S(1) line at $2.1218 \mu \mathrm{m}$ and images with the F215N filter give a continuum image. The flux-calibrated images were subtracted, following a scaling of the F215N image to account for the shape of the continuum.

High-dispersion, long-slit spectral observations were made of all four PPNe in the same $\mathrm{H}_{2}$ line using the near-IR echelle spectrograph Phoenix at the Gemini South or KPNO 4-m (for IRAS 17150) telescopes. The resolution is $\sim 50,000\left(\sim 6 \mathrm{~km} \mathrm{~s}^{-1}\right)$ with a slit width of $0.34^{\prime \prime}$ (Gemini-S) or $0.74^{\prime \prime}$ (KPNO). Spectra were obtained at several positions on the nebula, based upon the $\mathrm{H}_{2}$ images. From these, position-velocity (PV) diagrams were formed. 

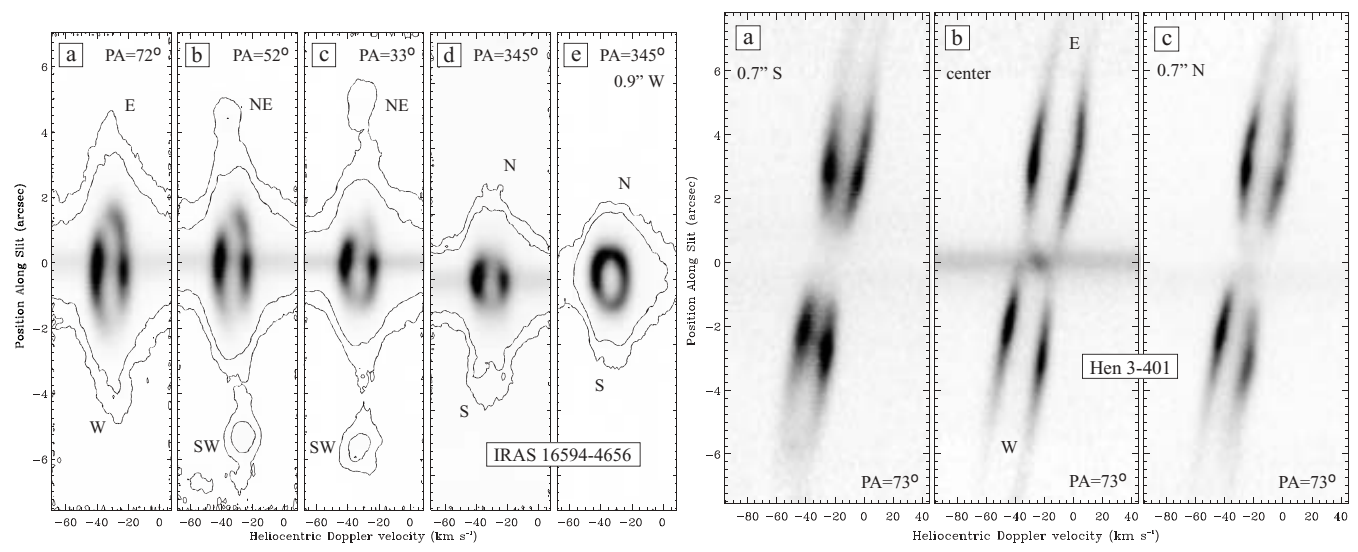

Figure 1. PV diagrams for (a) IRAS 16594-4655 and (b) Hen 3-401

\section{Results}

\subsection{IRAS 16594-4656 (Water Lily nebula)}

The PV diagram at each slit position shows a bright closed ellipse with variations in size and brightness (Fig. 1a). These are consistent with limb-brightened lobes. The axis of each shows very little tilt, implying that the lobes are in the plane of the sky. This is in contrast to the impression from the visible image that the nebula is at some intermediate orientation. The faint outer emission from the clumps does show some velocity difference between the $\mathrm{E}$ and $\mathrm{W}$ sides. These could be the result of ejections by precessing jets.

\subsection{Hen 3-401 (IRAS 10178-5958)}

The polar axis is obviously tilted from the plane of the sky, with the $\mathrm{W}$ lobe moving toward us (Fig. 1b). Velocities along the lobes are proportional to distance from the star (Hubble flow). The pairs of convex arcs represent emission from the front and back of slightly barrel-shaped lobes, which show an expansion velocity of $15 \mathrm{~km} \mathrm{~s}^{-1}$.

\subsection{Rob 22 (IRAS 10197-5750)}

The spectra of the nebula show very faint $\mathrm{H}_{2}$ emission from two hollow expanding lobes. The polar axis appears to be close to the plane of the sky, with the S lobe slightly blueshifted. The expansion speed in the $\mathrm{S}$ lobe is $\sim 15 \mathrm{~km} \mathrm{~s}^{-1}$. The $\mathrm{H}_{2}$ emission is relatively weak and the spectra are dominated by scattered continuum light.

\subsection{IRAS 17150-3224 (Cotton Candy nebula)}

The PV diagram shows that the two lobes have the same velocity and thus are in the plane of the sky. The $\mathrm{H}_{2}$ emission arises primarily at the ends of the lobes, with additional weak emission from an equatorial loop and an ear-shaped region outside the top lobe. The $\mathrm{H}_{2}$ line width and intensity are consistent with C-shocks.

\section{Acknowledgements}

Support from NASA through grants GO-07840.02-A, GO-07840.01-A, GO-09366.01-A, GO-09366.02-A, GO-09366.03-A, and HF-01166.01-A from the Space Telescope Institute, which is operated by AURA, is acknowledged, as is support to BJH from the NSF (0407087). 